\title{
Chingree: Grasshopper Plugin to Generate 2D Compositions and 3D Embodiments Inspired from Mondrian's Neo-plastic Compositions Using Shape Grammar
}

\author{
Joy Mondal \\ WEsearch lab | India | joy@wesearchlab.com
}

\begin{abstract}
The paper documents the development of algorithms (releasied as Grasshopper plugin Chingree) to generate 2D compositions and 3D embodiments inspired from the visual ethos of Mondrian's neo-plastic compositions. Based on the empirical analysis of the compositions; rectangles are sub-divided with lines parallel to $x$ and $y$ axes, followed by selection of few of the sub-divisions for colouring. The principles of sub-division and selection are applied in 3D. Volumes are sub-divided with planes parallel to $x y, y z$ and $z x$ planes. Few of the subdivisions are selected to be present (analogous to colouring in 2D). Shape grammar is used to control sub-division.
\end{abstract}

Keywords: Neo-plastic; Mondrian; Shape grammar; 3D massing; Sub-division.

\section{INTRODUCTION}

Art and architecture have a symbiotic relationship of inspiring and influencing each other. Architecture has often been a compositional element in paintings - for example, the cityscape as a container in Renaissance art. Additionally, architecture has been often used as contextual element and background for displays and installations - for example, the wrapping of Reichstag in Berlin by Christo- and Jeanne-Claude, and the Cloud Gate at AT\&T Plaza in Chicago by Anish Kapoor, respectively. Conversely, art movements and artists have shaped the visual language of architecture movements and architects. Few examples include Piet Mondrian's neo-plastic compositions' influence on Frank Lloyd Wright's philosophy of spatial configuration and Mies van der Rohe's plan of the Barcelona Pavilion (AI Saati, 1990), Sol LeWitt's sculptures' influence on Peter Eisenman's early house designs (Luscombe, 2014), and the influence of suprematist paintings of Kazimir Malevich on Zaha Hadid's early designs (Abdullah, Said, \& Ossen, 2013).

Architects' appropriation of paintings and sculptures into 3D compositions remains largely documented with texts that are more suggestive than prescriptive in nature (Abdullah et al., 2013; Al Saati, 1990; Collins, 1965; Luscombe, 2014; Scully, 1960). Consequently, other architects appropriate the appropriation. In contemporary times, particularly in Mondrian's case and to a large extent in the case of neo-plasticism, instead of translating the visual ethos of the compositions into 3D, the compositions are typically used on vertical surfaces as extruded embellishment or as wallpaper like surface mapping through colour treatment.

This paper presents methods to generate compositions similar to and 3D embodiments inspired by Mondrian's neo-plastic 2D compositions. Shape grammar is used to sub-divide planar closed curves and closed volumes by adding sub-dividing lines and sub-dividing planes on underlying line grid and plane grid respectively. The objective is to appropriate (or mimic) the visual ethos of the $2 \mathrm{D}$ compositions into $3 \mathrm{D}$ massing which can be subsequently used as a starting point for design articulation. The approach is an empirical interpretation of the visual structure (or style) of the compositions. It is not a definitive model for reproducing the compositions. The algorithms were investigated using custom C\# scripts on Grasshopper and have been subsequently released as a free plugin named Chingree.

In the context of this paper, visual ethos means the apparent characteristic(s) of the visual make up of an object. Visual ethos for a given object may vary from one interpretation to the other. For example, in the painting titled 'The Vase with Twelve Sunflowers' by Vincent Van Gogh, the varying radii in plan at different levels and the undulating curvature in elevation can be interpreted as alternative visual ethos of the flower vase. Appropriation shall mean the process of extrapolating the interpretation of visual ethos to generate a similar artefact. In the same example, the flower vase may be appropriated by lofting a surface through four circles of varying radii or by sweeping an undulated curve around a vertical axis. Different arrangements of different radii or different curvatures of the undulated curve shall produce visually 'similar' iterations of the flower vase.

The following sections elaborate the objectives, the analysis of the original compositions, its 2D appropriation, translation of the $2 \mathrm{D}$ ethos to $3 \mathrm{D}$, the procedure to generate 3D embodiments, and the Grasshopper components of Chingree.

\section{BACKGROUND}

The neo-plastic (literal translation: new plastic) paintings of Piet Mondrian are simple abstract compositions of horizontal and vertical black lines with red, yellow, blue 
and black rectangles on white and off-white canvases (Veen, 2017). These paintings are a departure from traditional content of subject, cultural references, representations and nature. The paintings do not use natural colors of green or brown, modulation in lightness, or sense of depth. Instead, the paintings exploit a vocabulary of pure abstraction by using line grids.

\section{MONDRIAN'S NEO-PLASTIC COMPOSITIONS AND ARCHITECTURE}

Piet Mondrian was influenced by Frank Lloyd Wright's work, specifically the interior of Unity Temple. Mondrian's neo-plastic compositions later influenced Wright's design of the Falling Water (Al Saati, 1990). The International Style of the $20 \mathrm{~s}$ and $30 \mathrm{~s}$ got its inspiration from Mondrian's principles of simple horizontal and vertical intersecting lines and asymmetric visual weight (Scully, 1960). Mondrian's influence is also noticeable in some of Mies van der Rohe's works - the plans of Barcelona Pavilion and Brick Country House (Collins, 1965). Mondrian's principles of plasticity allowed the architects to play with abstraction of mass. Mondrian's planes and grids are not only visually similar to the operative design techniques of modernism, but also compliment the industrial production of architecture.

\section{PREVIOUS WORKS ON GENERATION OF NEO- PLASTIC COMPOSITIONS}

Different studies have used shape grammar, genetic representation and machine learning to generate neoplastic compositions. Shape grammar has been successfully used to sequentially sub-divide a rectangle to appropriate compositions of other neo-plastic artists like Georges Vantongerloo and Fritz Glarner (Knight, 1989). However, in the research, 3D embodiments of the compositions of the artists have not been subsequently generated by using the principle of sub-dividing shape grammar. Genetic representation has been used to crossbreed Mondrian's paintings and Wright's window design for Hollyhock House (Schnier \& Gero, 1998). This process produces 2D output with incoherent lines. Additionally, the 3D embodiment generated in this process maps $2 \mathrm{D}$ output on the surface of a cube. Surface mapping fails to introduce overhangs and porosity which are key elements of Wright's and Rohe's appropriations of Mondrian's paintings. Machine learning has been used by training pattern recognition algorithm with positive and negative examples of neo-plastic compositions to generate matrices like aspect ratio, number of lines, visual weightage of colours etc. (Andrzejewski, Stork, Zhu, \& Spronk, 2010). The trained model generates compositions with far fewer number of split lines compared to the original compositions. The research in this paper deliberately takes a departure from black box algorithms. It uses a series of steps which can be executed by coding, explicit modelling or manual drawing to make the process similar to the procedural nature of early stage design exploration.

\section{OBJECTIVES}

The objectives of the research with the aim to appropriate the visual ethos of the 2D compositions into $3 \mathrm{D}$ embodiments are as following -
1. To conceive a sequentiall algorithm using shape grammar which can also be executed by explicit modeling or manual drawing;

2. To extract the morphologicial components of the $2 \mathrm{D}$ compositions; and

3. To re-represent the extracted morphological components to generate $3 \mathrm{D}$ embodiments.

\section{TOOLS USED}

The algorithms presented in this paper have been developed through visual scripting on Grasshopper. Subsequently, a Grasshopper plugin named 'Chingree' has been developed for ease of use and distribution. Chingree can be downloaded from the food4rhino website at www.food4rhino.com/app/chingree.

\section{MORPHOLOGICAL ANALYSIS OF MONDRIAN'S NEO-PLASTIC COMPOSITIONS}

\section{PAINTINGS FOR ANALYSIS}

Mondrian's 45 classical neo-plastic compositions (Figure 1) are used for analysis to extract an interpretation of visual ethos. The compositions were made between 1920 and 1943; with widths of average $59.6 \mathrm{~cm}$, minimum 32.1 $\mathrm{cm}$ and maximum $120.0 \mathrm{~cm}$, and heights of average 68.7 $\mathrm{cm}$, minimum $40.0 \mathrm{~cm}$ and maximum $145.2 \mathrm{~cm}$. Compositions with diagonal lines or diamond canvases are not included in the analysis.

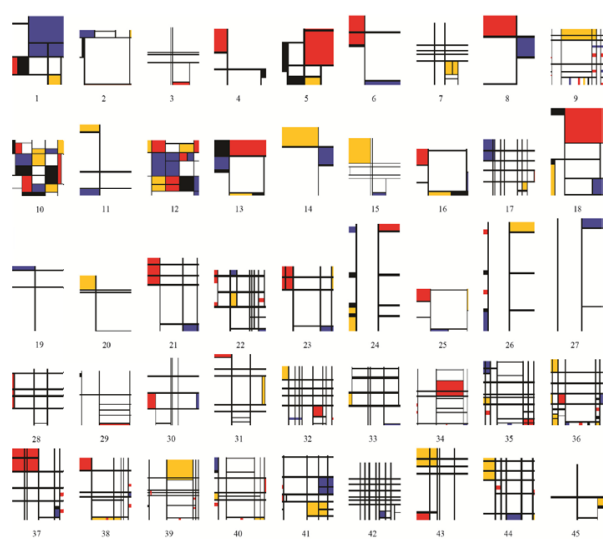

Figure 1: Digital images of the 45 compositions used for empirical anialysis.

\section{ASSUMPTIONS AND EXCLUSIONS}

The goal of the analysis is to appropriate the visual ethos of (and not reproduce) the compositions. Consequently, the following assumptions and exclusions are made -

1. Subtleties in colour variation of the rectangles and variations in stroke width of lines are ignored; and

2. White and off-white rectangles are assumed to be colourless. 


\section{EMPIRICAL OBSERVATIONS}

Empirical analysis of the structure of Mondrian's 45 neoplastic compositions (Figure 1) - used both as a context and content for developing the shape grammar rule-set leads to the following observations -

1. The canvases are rectangular in shape (Figure 2);

2. The rectangles vary in aspect ratio;

3. The canvases are sub-divided with lines parallel to $x$ axis and $y$-axis (Figure 2);

4. The lines parallel to x-axis are of two kinds - edge to edge and split;

5. The lines parallel to $y$-axis are of two kinds - edge to edge and split;

6. The end points of the edge to edge lines parallel to $x$ axis always touch opposite canvas edges parallel to $y$ axis;

7. The end points of the edge to edge lines parallel to $y$ axis always touch opposite canvas edges parallel to $\mathrm{x}$ axis;

8. The end points of the split lines parallel to x-axis exhibit one of the following four conditions -

8.1. Touch edge to edge lines parallel to $y$-axis on both sides:

8.2. Touch split lines parallel to $y$-axis on both sides;

8.3. Touch edge to edge line parallel to $y$-axis on one side and split line parallel to $y$-axis on the other side; or

8.4. Touch edge to edge line parallel to $y$-axis on one side and one of the canvas edges parallel to $y$ axis on the other side;

9. The end points of the split lines parallel to $y$-axis exhibit one of the following four conditions -

9.1. Touch edge to edge lines parallel to $x$-axis on both sides;

9.2. Touch split lines parallel to $x$-axis on both sides;

9.3. Touch edge to edge line parallel to $x$-axis on one side and split line parallel to $x$-axis on the other side; or

9.4. Touch edge to edge line parallel to $x$-axis on one side and one of the canvas edges parallel to $x$ axis on the other side;

10. Few of the sub-divisions are selected for colouring (Figure 2); and

11. Colours used on the sub-divisions are red, blue, yellow and black (Figure 2).
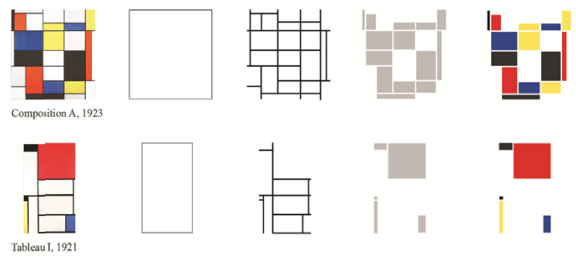

Figure 2: Morphological components of the 2D compositions canvas proportion, sub-dividing lines, selected sub-divisions and colouring of the selected sub-divisions; demonstrated through two Mondrian compositions.

\section{D APPROPRIATION WITH SHAPE GRAMMAR}

The empirical observations show that the canvases are sub-divided with four kinds of lines. On sub-division, few of the canvas sub-divisions are selected for colouring. To make the process procedural (and consequently, iterative) the sub-dividing lines on the compositions are assumed to be added sequentially. Colours are taken to imply presence of the sub-divisions. White and off-white (colourless) are taken to imply absence of the subdivisions. The compositions can be appropriated by undertaking the following steps -

1. Start with a rectangle;

2. Overlay an orthogonal grid on the rectangle to act as candidate sub-dividing lines;

3. Add a combination of the four kinds of sub-dividing lines (edge to edge and split lines parallel to $x$-axis and $y$-axis) on the rectangle using the orthogonal grid as guidelines;

4. Select sub-divisions of the rectangle for colouring; and

5. Colour the selected sub-divisions with red, blue, yellow and black.

The sub-division of the rectangle with lines (step 3) is controlled by using a shape grammar rule sequence as explained in the following sub-section.

\section{D SHAPE GRAMMAR RULE-SET}

Shape grammar has traditionally been used to model conceptual massing (Stiny, 1980) and appropriate stylistic massing (Benros, Hanna, \& Duarte, 2014). Shape grammar consists of shape rules that define how an existing shape can be transformed. Rules are typically defined in the form of two shapes connected by an arrow: $A \rightarrow B$, where $A$ and $B$ are shapes. The shape grammar used in this paper adds sub-dividing lines and planes on underlying grids of candidate sub-dividing lines and planes for 2D appropriation and 3D generation respectively. Once the underlying grid is defined, the generation of the $2 \mathrm{D}$ appropriations and $3 \mathrm{D}$ embodiments are controlled by the order of the rules. Consequently, the focus is not so much on the left hand side $(A)$ and the right hand side (B) of the rule(s) as it is on the rule sequence (and thereby the order of rules in the rule sequence) that acts as a string-based generation guide. 
To illustrate how the rules add different sub-dividing lines or planes, the characters identifying the rules (A, B, C etc.) are written on the arrow between the left hand side and the right hand side (Figure 3 ). The rule characters are not to be confused with the conventional notations of left hand side shape $A$ and right side shape $B$.

The Mondrian compositions have four kinds of subdividing lines (edge to edge sub-dividing lines parallel to $x$-axis and $y$-axis and split sub-dividing lines parallel to $x$ axis and $y$-axis). Therefore, four kinds of shape grammar rules corresponding to the four kinds of sub-dividing lines are used for $2 \mathrm{D}$ appropriation. Rules $\mathrm{A}$ and $\mathrm{B}$ add edge to edge sub-dividing lines parallel to $x$-axis and $y$-axis respectively, and rules $C$ and $D$ add split sub-dividing lines parallel to $x$-axis and $y$-axis respectively. Figure 3 shows the four rules with the example of a $5 \times 5$ candidate sub-dividing grid overlaid on a square canvas. The four rules are elaborated as following -

1. Rule A (edge to edge line parallel to $x$-axis): Adds a sub-dividing line parallel to $x$-axis on the rectangle from one of the candidate sub-dividing lines (from the grid) parallel to x-axis. In the example in Figure 3, subdividing line for rule A may be added on any of the five candidate sub-dividing lines parallel to $\mathrm{x}$-axis.

2. Rule B (edge to edge line parallel to $y$-axis): Adds a sub-dividing line parallel to $y$-axis on the rectangle from one of the candidate sub-dividing lines (from the grid) parallel to $y$-axis. In the example in Figure 3, subdividing line for rule $B$ may be added on any of the five candidate sub-dividing lines parallel to $y$-axis

3. Rule C (split line parallel to $x$-axis): Selects one of the candidate sub-dividing lines parallel to $x$-axis from the grid and splits it by edge to edge and split lines parallel to $y$-axis (added on the rectangle by preceding Rule Bs and Ds). One of the split members of the selected candidate sub-dividing line is added on the rectangle as a split line. In the example shown in Figure 3 , sub-dividing line for rule $\mathrm{C}$ may be added as any one of the fifteen split lines parallel to x-axis. On the execution of this rule, the example rule sequence will be BBC.

4. Rule D (split line parallel to $y$-axis): Selects one of the candidate sub-dividine $\mathrm{g}$ lines parallel to $\mathrm{y}$-axis from the grid and splits it by edge to edge and split lines parallel to $\mathrm{x}$-axis (added on the rectangle by preceding Rule As and Cs). One of the split members of the selected candidate sub-dividing line is added on the rectangle as a split line. In the example shown in Figure 3, sub-dividing line for rule D may be added as any one of the fifteen split lines parallel to $y$-axis. On the execution of this rule, the example rule sequence will be AAD.

At the conclusion of every rule in a rule sequence, the selected candidate sub-dividing line for the rule is deleted from the grid to avoid overlap of sub-dividing lines. In case of rules $C$ and $D$, splitting the selected candidate subdividing lines by sub-dividing lines of preceding rules in the opposite direction ensures satisfaction of one of the four end conditions of split lines.
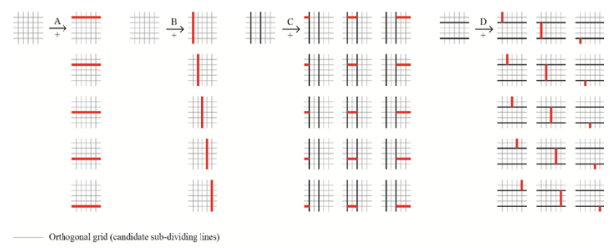

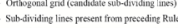

Figure 3: Shape grammar rulle-set to appropriate 2D compositions on a square canvas with $5 \times 5$ candidate sub-dividing grid; all possible options for adding sub-dividing lines based on the left hand sides and rules are shown.

It is to be noted that since the underlying grid of candidate sub-dividing lines are bound to the extents of the canvas, the edge to edge and split sub-dividing lines automatically get scaled with any change in the aspect ratio or size of the canvas. Distinction is made lbetween sub-dividing lines parallel to $x$-axis and $y$-axis (instead of using a rule to rotate sub-dividing lines) to have a direct injective control on the number of sub-dividing lines in the appropriation. Consequently, for a rule sequence $A B C D$, the sub-division lines added on the rectangle will correspond to rule $A$, rule $B$, rule $C$ and rule $D$ i.e. one edge to edge sub-dividing line parallel to $x$-axis, followed by one edge to edge subdividing line parallel to $y$-axis, followed by one split subdividing line parallel to $x$-axis and concluded by one split sub-dividing line parallel $y$-axis.

\section{D SEQUENTIAL PROCESS}

Figure 4 shows various sequential sub-divisions of a square by using AABBDC as the rule sequence. Different sub-dividing lines from the grid are added to the square with the addition of each rule from the rule sequence. Subsequently, $4 / 11$ of the sub-divisions are selected for colouring.

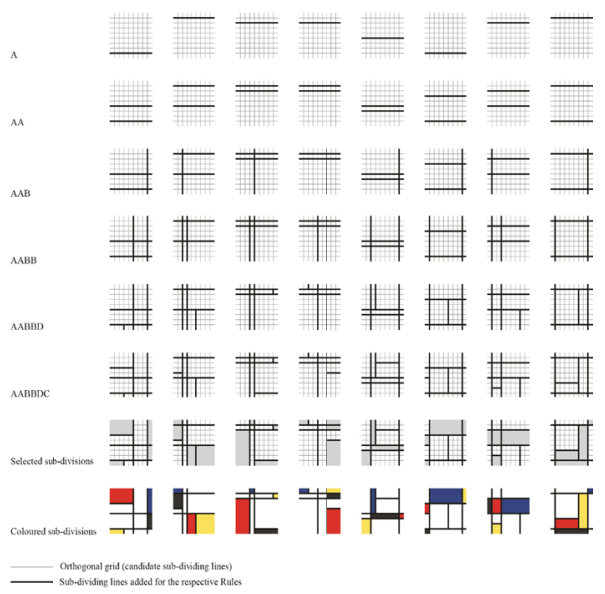

Figure 4: Sequential process of generation of 2D compositions on a square using $A A B B D C$ as the rule sequence and $4 / 11$ subdivisions selected for colouring.

\section{D VARIABLES}

The variables in the algorithm for appropriating 2D compositions are as following - 
1. The (aspect ratio of) canvas;

2. Number of candidate grid lines in $\mathrm{x}$ and $\mathrm{y}$ directions;

3. Shape grammar rule sequence;

4. Order of selection of grid lines according to the rule sequence;

5. Number (or percentage) of sub-divisions to be selected (retained);

6. Order of selection of sub-divisions to be selected (retained); and

7. Order of distribution of the four colours on the selected sub-divisions.

Random functions are used in the examples in this paper and in the components of Chingree for variables 4, 6 and 7. One may add custom conditions for retention of subdivisions.

\section{GENERATING 3D EMBODIMENTS}

3D embodiments are also generated based on the empirical analysis of the original compositions. The principles of sub-dividing a geometry and subsequent selection of the sub-divisions for retention are applied in $3 D$. The following sections explain the translation of the elements from $2 D$ to $3 D$, the $3 D$ shape grammar rule-set and the $3 \mathrm{D}$ variables.

\section{D TO 3D TRANSLATION}

When moved from $2 D$ to $3 D$, the initial geometry changes from canvas (2D) to various input primitives (3D), the subdividing agents change from lines (1D) to planes (2D), and sub-divided components change from rectangles (2D) to cuboids (3D). Consequently, the 2 axes ( $x$ and $y$ ) change to 3 planes ( $x y, y z$ and $z x$ ). Presence and absence of colour is taken as a morphological tool to determine the presence and absence of the sub-divisions. The selected sub-divisions are not literally coloured (Figure 5).
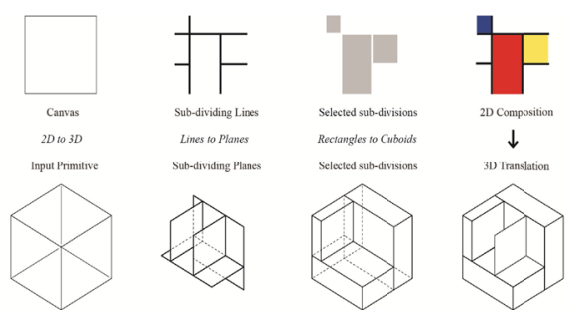

Figure 5: 2D to $3 \mathrm{D}$ translation of the morphological components.

Thus, similar to the $2 \mathrm{D}$ appropriations, 3D embodiments can be generated by undertaking the following steps -

1. Start with a $3 D$ input primitive;

2. Overlay an orthogonal grid in the input primitive to act as candidate sub-dividing planes;
3. Add a combination of six kinds of sub-dividing planes (face to face planes analogous to edge to edge lines and split planes analogous to split lines, parallel to $x y$, $y z$ and $z x$ planes) in the input primitive using the orthogonal grid as guideline; and

4. Select sub-divisions of the input primitive for presence (analogous to colouring).

The sub-division of the input primitive with planes (step 3) is executed by using shape grammar as explained in the following sub-section.

\section{D SHAPE GRAMMAR RULE-SET}

Similar to the $2 \mathrm{D}$ appropriations, shape grammar is used for sequential sub-division of the input primitives. Similar to the edge to edge and split sub-dividing lines in the case of $2 \mathrm{D}$ appropriation, face to face and split sub-dividing planes are added in the input primitives. Rules A, B and C add $a$ face to face sub-dividing plane parallel to the $x y, y z$ and $z x$ planes respectively, and rules $D, E$ and $F$ add a split sub-dividing plane parallel to the $x y, y z$ and $z x$ planes respectively. Figure 6 shows the six rules with the example of a $5 \times 5 \times 5$ candidate sub-dividing grid overlaid in a cube. The six rules are elaborated are as following -

1. Rule A (face to face plane parallel to xy-plane): Adds a sub-dividing plane parallel to $x y$-plane in the input primitive from one of the candidate sub-dividing planes parallel to $x y$-plane of the grid. In the example shown in Figure 6, sub-dividing plane for rule $A$ may be added on any of the five candidate sub-dividing planes parallel to xy-plane.

2. Rule B (face to face plane parallel to yz-plane): Adds a sub-dividing plane parallel to yz-plane in the input primitive from one of the candidate sub-dividing planes parallel to yz-plane of the grid. In the example shown in Figure 6 , sub-dividing plane for rule $B$ may be added on any of the five candidate sub-dividing planes parallel to yz-plane.

3. Rule C (face to face plane parallel to zx-plane): Adds a sub-dividing plane parallel to $\mathrm{zx}$-plane in the input primitive from one of the candidate sub-dividing planes parallel to zx-plane of the grid. In the example shown in Figure 6 , sub-dividing plane for rule $\mathrm{C}$ may be added on any of the five candidate sub-dividing planes parallel to zx-plane.

4. Rule D (split plane parallel to xy-plane): Selects one of the candidate sub-dividing planes parallel to $x y$-plane from the grid and splits it by face to face and split planes parallel to $\mathrm{yx}$ and $\mathrm{zx}$ planes (added by preceding Rule Bs, Cs, Es and Fs). One of the split members of the selected candidate sub-dividing plane is added in the input primitive. In the example shown in Figure 6, sub-dividing plane for rule $D$ may be added as any one of the twenty split planes parallel to $x y$-plane. On the execution of this rule, the example rule sequence will be $B C D$.

5. Rule E (split plane parallel to yz-plane): Selects one of the candidate sub-dividing planes parallel to yz-plane from the grid and splits it by face to face and split planes parallel to $x y$ and $z x$ planes (added by 
preceding Rule As, Cs, Ds and Fs). One of the split members of the selected candidate sub-dividing plane is added in the input primitive. In the example shown in Figure 6, sub-dividing plane for rule $E$ may be added as any one of the twenty split planes parallel to yz-plane. On the execution of this rule, the example rule sequence will be ACE.

6. Rule F (split plane parallel to zx-plane): Selects one of the candidate sub-dividing planes parallel to zx-plane from the grid and splits it by face to face and split planes parallel to $x y$ and $y z$ planes (added by preceding Rule As, Bs, Ds and Es). One of the split members of the selected candidate sub-dividing plane is added in the input primitive. In the example shown in Figure 6 , sub-dividing plane for rule $F$ may be added as any one of the twenty split planes parallel to zx-plane. On the execution of this rule, the example rule sequence will be $\mathrm{ABF}$.

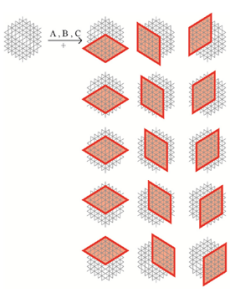

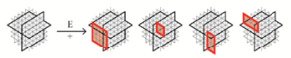
1 196 18

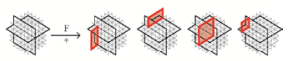
辣 ? \% $\rightarrow>$

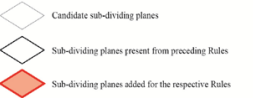

Figure 6: Shape grammar rule-set to generate 3D embodiments using a cube with $5 \times 5 \times 5$ candidate sub-dividing grid; all possible options for adding sub-dividing planes based on the left hand sides and rules are shown.

At the conclusion of every rule, the selected candidate sub-dividing plane for the rule is deleted from the grid to avoid overlap of sub-dividing planes.

\section{D SEQUENTIAL PROCESS}

Figure 7 shows various sequential sub-divisions of a cube by using $A A B B C C D E F$ as the rule sequence. Different sub-dividing planes are added to the cube with the addition of each rule from the rule sequence. Subsequently, $18 / 30$ of the sub-divisions are selected for retention (presence).

\section{D VARIABLES}

The variables in the algorithm to generate $3 \mathrm{D}$ embodiments are as following -

1. Input primitive shape;
2. Number of candidate planes parallel to $x y, y z$ and $z x$ planes;

\section{Shape grammar rule sequence;}

4. Order of selection of candidate planes according to the rule sequence;

5. Number (or percentage) of sub-divisions to be selected (retained); and

6. Order of selection of sub-divisions to be selected (retained).

Random functions are used in the examples in this paper and in the components of Chingree to generate the orders of selection as mentioned in 4 and 6 . One may add custom conditions for retention of sub-divisions.

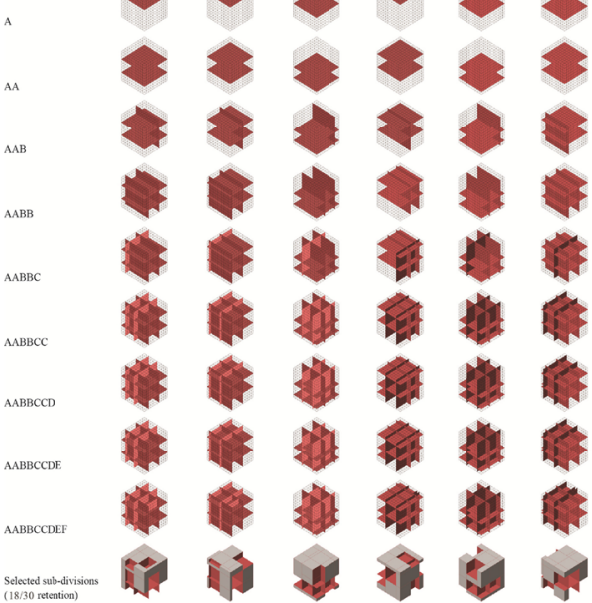

Figure 7: Sequential process of generation of $3 D$ embodiments with a cube using AABBCCDEF as the rule sequence and 18/30 selection of sub-divisions.

\section{CHINGREE GRASSHOPPER PLUGIN}

The algorithms discussed in this paper are released as a free Grasshopper plugin - Chingree - which uses five custom components to generate the $2 \mathrm{D}$ compositions and 3D embodiments. Chingree uses curve and brep representations, thereby allowing the user to generate $2 \mathrm{D}$ compositions and 3D embodiments using non-orthogonal grids, and non-orthogonal canvas shapes and volumes as well. Figure 8 shows a screenshot of the five components along with dummy scripts to generate $2 \mathrm{D}$ appropriations and 3D embodiments. The components are briefly described as following -

1. 2D Grid generator: Generates an orthogonal $2 \mathrm{D}$ grid of candidate sub-dividing lines for $2 \mathrm{D}$ appropriation by dividing an input closed curve. The numbers of candidate sub-dividing lines parallel to $x$ and $y$ are also variables. 
2. 2D Appropriation generator: Generates the geometry of $2 \mathrm{D}$ appropriations of Mondrian paintings based on the algorithm discussed in this paper. The inputs include all the 2D variables except the order of distribution of colours. The inputs for candidate subdividing lines may be taken from the output of the 2D Grid generator component. One may also use nonorthogonal custom candidate sub-dividing lines or curves of higher degree.

3. 3D Grid generator: Generates an orthogonal 3D grid of candidate sub-dividing planes for generating 3D embodiments by dividing an input closed poly-surface. The numbers of candidate sub-dividing planes parallel to $x y, y z$ and $z x$ are also variables.

4. 3D Embodiment generator: Generates the geometry of 3D embodiments mimicking the visual ethos of Mondrian paintings based on the algorithm discussed in this paper. The inputs include all the $3 \mathrm{D}$ variables. The inputs for candidate sub-dividing planes may be taken from the output of the 3D Grid generator component. One may also use non-orthogonal custom candidate sub-dividing planes or surfaces of higher degree.

5. Colour distributor: Separates the canvas divisions (taken from the output of 2D Appropriation generator) or volume divisions (taken from the output of $3 \mathrm{D}$ Embodiment generator) into four different lists for colouring with Mondrian red, blue, yellow and black. Additionally, generates the corresponding list of repeated colours. The inputs include a numerical seed to randomise the order of distribution of colours.

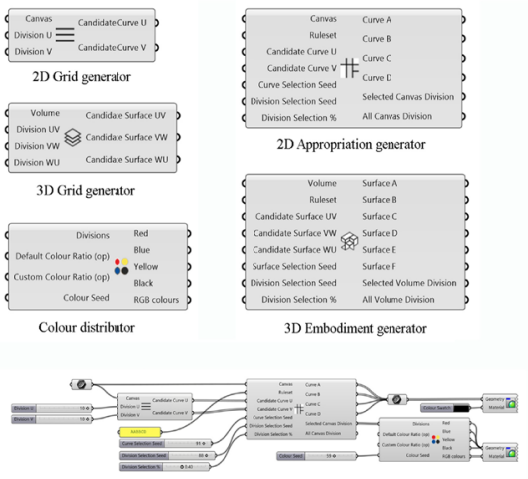

2D Appropriation generator

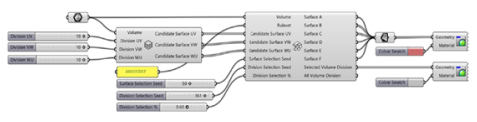

3D Embodiment generator

Figure 8: The five components of Chingree, and dummy scripts for $2 \mathrm{D}$ appropriation and 3D embodiment generation.

\section{RESULTS}

Figure 9 shows variations of 3D embodiments of a cube as input primitive. Figure 10 shows variations of $3 \mathrm{D}$ embodiments of two types of input primitives - cuboid vertical and cuboid union. The same logic can be applied to non-orthogonal input primitives consisting of single curved surfaces like cylinder, double curved surfaces like spheres or any other free-form closed poly-surface. These may be sub-divided using planes parallel to the orthogonal planes or by custom surfaces of any orientation and degree (order). For example, Figure 11 shows variations of $3 \mathrm{D}$ embodiments of a tube quadrant using $A B B B C C D E E F$ as the rule sequence. Custom candidate sub-dividing surfaces are used to generate the embodiments. The variations have the same selected sub-dividing surfaces, two sets of sub-division selection percentages $(50 \%$ and $70 \%)$ and different order of selection of the sub-divisions.

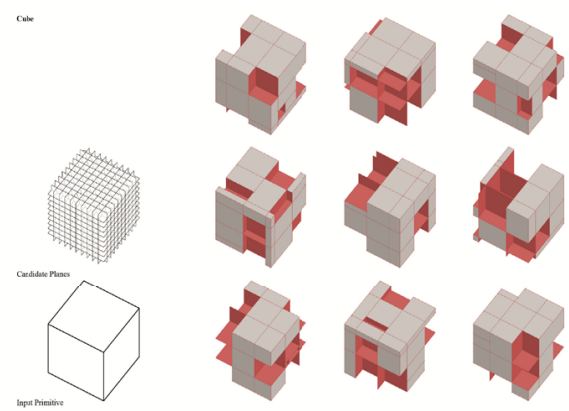

Figure 9: Variations of 3D embodiments of a cube as input primitive.

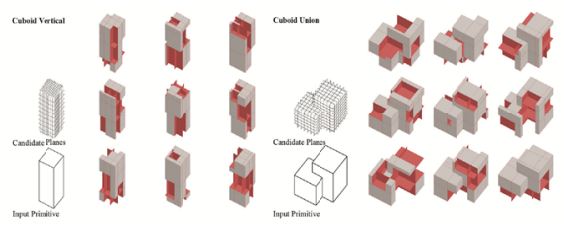

Figure 10: Variations of 3D embodiments of two types of input primitives -1 ) cuboid vertic:al and 2) cuboid union.

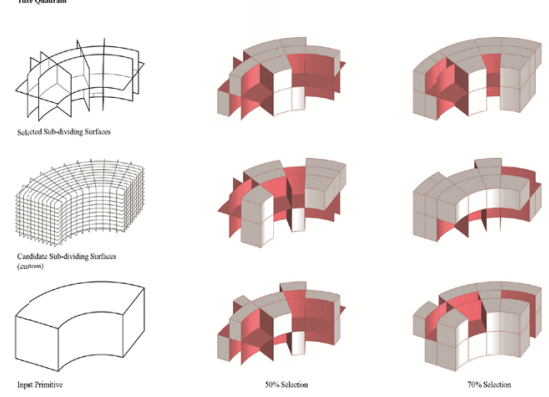

Figure 11: Variations of 3D embıdiments of a tube quadrant using $A B B B C C D E E F$ as rule sequence generated with custom candidate sub-dividing surfaces while varying sub-division selection $(50 \%$ and $70 \%)$.

The flattened elevations (Figure 12) of 3D embodiments appropriate the ethos of the Neo-plastic compositions. 
The procedural nature of the method can be extended to deconstruct any architectural typology to generate infinite variations. These can act as the starting points for finer articulation of the geometry in the design development phase.
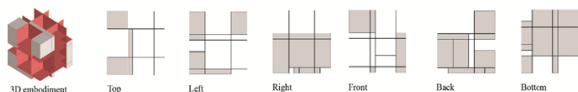

Figure 12: Flattened elevations of 3D embodiments appropriate the visual ethos of $2 \mathrm{D}$ compositions.

\section{CONCLUSION}

The paper has presented the sequential methods used in Chingree to generate Mondrian like 2D compositions and Mondrian inspired 3D neo-plastic embodiments. Presence and absence of colour is used as a morphological tool to determine the presence and absence of the sub-divisions. When $2 \mathrm{D}$ is translated to $3 \mathrm{D}$, sub-division by lines changes to sub-division by planes. 3D primitive shapes are sub-divided with two kinds of planes (face to face and split) parallel to $x y, y z$ and zx planes, and subsequently few of the sub-divisions are selected to be present (analogous to colouring in 2D). The use of shape grammar to sub-divide geometry makes the process operative and iterative in nature, similar to the early stage rapid design exploration. Future additions shall include the development of an evaluative model to assess the visual similarity of the Chingree generated 2D compositions and the $3 \mathrm{D}$ embodiments to the original paintings.

\section{ACKNOWLEDGMENTS}

The author will like to thank Somen Chakraborty, Professor Emeritus, Jadavpur University and Debasish Das, Associate Professor, Jadavpur University for their guidance and comments on the research methodology.

\section{REFERENCES}

Abdullah, A., Said, I., \& Ossen, D. (2013). Zaha Hadid's Techniques of Architectural Form-Making. Open Journal of Architectural Design, 1(1), 1-9.

Al Saati, A. (1990). Mondrian: Neoplasticism and its Influences in Architecture. Middle East Technical University Journal of the Faculty of Architecture, 10(1-2), 63-74.

Andrzejewski, D., Stork, D. G., Zhu, X., \& Spronk, R. (2010). Inferring compositional style in the neo-plastic paintings of Piet Mondrian by machine learning. In D. G. Stork, J. Coddington, \& A. Bentkowska-Kafel (Eds.), Proceedings of SPIE: Vol. 7531. Computer Vision and Image Analysis of Art.

Benros, D., Hanna, S., \& Duarte, J. P. (2014). A Generic Shape Grammar for the Palladian Villa, Malagueira House, and Prairie House. In J. S. Gero (Ed.), Design Computing and Cognition (pp 321-340). Dordrecht: Springer.

Collins, P. (1965). Changing Ideals in Modern Architecture, 17501950 (pp 279-280). London: Faber and Faber.

Knight, T. W. (1989). Transformations of De Stijl Art: The Paintings of Georges Vantongerloo and Fritz Glarner. Environment and Planning B: Planning and Design, 16(1), 51-98

Luscombe, D. (2014). Architectural iconcepts in Peter Eisenman's axonometric drawings of House VI. The Journal of Architecture, 19(4), 560-611.

Schnier, T., \& Gero, J. S. (1998). From Frank Lloyd Wright to Mondrian: Transforming evolving representations. In I. C. Parmee (Ed.), Adaptive Computing in Design and Manufacture (pp 207-219). London: Springer.

Scully, V. (1960). Frank Lloyd Wright (Masters of World Architecture). New York: George Braziller.

Stiny, G. (1980). Introduction to Shape and Shape grammars. Environment and Planning B: Planning and Design, 7(3), 343-351.

Veen, L. (2017). Piet Mondrian on the Principles of NeoPlasticism. International Journall of Art and Art History, 5(2), 1-12. 